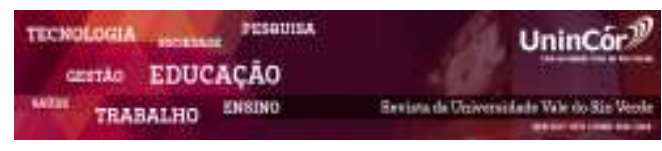

Revista da Universidade Vale do Rio Verde ISSN: 1517-0276 / EISSN: 2236-5362 Vol. 16 | n. 1 | Ano 2018

Iracy Marles Godim Fabiano Universidade Presbiteriana Mackenzie ira_mg@hotmail.com

Daniela Maria Alves Chaud Universidade Presbiteriana Mackenzie daniela.chaud@mackenzie.br

Edeli Simioni de Abreu Universidade Presbiteriana Mackenzie edelisabreu@gmail.com

\section{CONSUMO DE ALIMENTOS SEGUNDO O GRAU DE PROCESSAMENTO POR CRIANÇAS DE ESCOLAS PRIVADAS DA REGIÃO METROPOLITANA DE SÃO PAULO}

\section{RESUMO}

Objetivo: Investigar o consumo de alimentos segundo grau de processamento por crianças. Método: Trata-se de um estudo exploratório transversal, que foi realizado com crianças de 2 a 7 anos frequentadoras de escolas privadas da região metropolitana de São Paulo. Os dados foram coletados através de questionário do SISVAN (2015). Resultados: Participaram 70 crianças, sendo $55,71 \%$ meninas. Os marcadores do consumo alimentar avaliados revelam que a maioria das crianças consome feijão (87\%); verduras e/ou legumes (70\%) e frutas (62\%), fato este considerado como positivo por serem alimentos fontes de vitaminas, minerais e fibras alimentares. Foram observados fatores negativos quanto ao consumo elevado de embutidos (51\%); macarrão instantâneo (51\%); salgadinhos e biscoitos salgados $(51 \%)$, podendo ser considerados prejudiciais se consumidos em excesso por serem ricos em gorduras e sódio. $\mathrm{O}$ consumo de bebidas adoçadas (81\%) e biscoitos doces, guloseimas e gelatina (77\%) foi considerado preocupante devido à alta concentração de açúcares simples e gorduras presentes nestes alimentos que podem causar problemas de saúde já nessa tenra idade. Conclusão: A alimentação das crianças avaliadas em relação aos alimentos in natura e minimamente processados pode ser considerada satisfatória; contudo, há que se considerar que os resultados ora apresentados refletem o consumo do dia anterior e que o consumo de alimentos processados e ultraprocessados foram considerados expressivos, sendo este um fator negativo tendo em vista suas características de baixa densidade nutricional e elevados componentes deletérios cujo consumo elevado pode apresentar repercussões para a saúde da criança.

Palavras-chave: Alimentação infantil. Consumo alimentar. Processamento de alimentos. Registros de dieta.

\section{FOOD CONSUMPTION ACCORDING TO THE DEGREE OF PROCESSING BY CHILDREN OF PRIVATE SCHOOLS IN THE METROPOLITAN REGION OF SÃO PAULO}


consumed beans $(87 \%)$; vegetables $(70 \%)$ and fruits (62\%), which is considered positive by being food sources of vitamins, minerals and dietary fiber. Negative factors were observed regarding as the high consumption of sausages $(51 \%)$; instant noodles $(51 \%)$; salty snacks and crackers $(51 \%)$, which can be considered harmful if consumed in excess for being rich in fats and sodium. The consumption of sweetened beverages $(81 \%)$ and sweet biscuits, candies and gelatine $(77 \%)$ was considered worrisome due to the high concentration of simple sugars and fats present in these foods that can cause health problems even at that young age. Conclusion: Feeding children evaluated in relation to fresh and processed foods can be considered satisfactory; However, it should be considered that the results presented here reflect the consumption of the previous day and that the consumption of processed and ultraprocessed foods were considered as expressive, being this a negative factor in view of its characteristics of low nutritional density and high deleterious components whose consumption May have repercussions on the health of the child.

Keywords: Child nutrition. Food consumption. Food handling. Diet Records. Nutritional transition.

Recebido em: 11/12/2016 - Aprovado em: 18/01/2018 - Disponibilizado em: 15/07/2018

\section{INTRODUÇÃO}

A alimentação humana, além de se referir à ingestão de alimentos necessária para a obtenção de nutrientes, também remete às características envolvidas no consumo, influenciando na saúde e bem-estar do indivíduo e envolvendo aspectos culturais e sociais das práticas alimentares (ARNEIRO, 2005; BRASIL, 2014; GALLIAN, 2007).

Nas últimas décadas houve um crescimento no consumo em excesso de produtos industrializados em substituição aos alimentos in natura, trazendo aos países em desenvolvimento uma inversão epidemiológica caracterizada pela ascensão de doenças crônicas não transmissíveis (DCNT), como obesidade, diabetes, hipercolesterolemia e hipertensão arterial, diagnosticadas cada vez mais precocemente (AQUINO et al., 2002).
Atualmente, observa-se que os indivíduos passam a consumir alimentos e/ou preparações sem que o nutriente principal seja o determinante na escolha, consequência de uma manobra explorada pelo comércio e indústria alimentícia que produzem, cada vez mais, alimentos práticos, palatáveis, duráveis e mais atrativos para a população (BIELEMANN et al., 2015). De acordo com Martins et al. (2013), a classificação desses alimentos quanto ao grau de processamento é relevante para o enfrentamento da obesidade e outras doenças crônicas.

A Classificação NOVA avalia processos físicos, biológicos e químicos que acometem os alimentos depois da colheita, antes de serem submetidos ao processo culinário (MONTEIRO et al., 2016). Divide-os em 4 grupos, sendo estes inseridos no novo Guia Alimentar Para a População Brasileira (BRASIL, 2014): in natura, 
minimamente processados, processados e ultraprocessados.

Os produtos ultraprocessados são essencialmente formulações da indústria com um número elevado de ingredientes, caracterizando a dieta de quem os consome como excessivas em sal, açúcar, óleos, gorduras e substâncias de uso industrial, cuja função é prolongar a validade destes alimentos ou melhorar suas características organolépticas (BARALDI et al., 2015; BRASIL, 2014; MONTEIRO et al., 2015).

De tal forma, torna-se mais frequente a preocupação em reduzir o consumo de alimentos processados e ultraprocessados e incentivar uma dieta equilibrada composta por: alimentos in natura, obtidos diretamente de plantas ou animais; e alimentos minimamente processados, submetidos a alterações mínimas, que incluem grãos em forma de farinhas, raízes e tubérculos lavados, cortes de carne resfriados ou congelados e leite pasteurizado, além de óleos, gorduras, açúcar e sal (BRASIL, 2014).

$\mathrm{Na}$ infância, fornecer uma alimentação adequada é essencial e determinante nas condições de saúde do indivíduo e propiciar um crescimento e desenvolvimento adequados (AQUINO et al., 2002). Deve ser equilibrada em relação ao valor energético e rica em proteínas e micronutrientes, em especial o ferro, o zinco, o cálcio, a vitamina $\mathrm{A}$, a vitamina $\mathrm{C}$ e os folatos, evitando-se o uso excessivo de sal e os alimentos fornecidos estando em quantidade, qualidade, variedade, moderação e harmonia corretas (BRASIL, 2007; WHO, 2000). A faixa etária pré-escolar demanda maior atenção por ser um período de crescimento lento, porém contínuo, de alta vulnerabilidade e susceptibilidade à má nutrição (OLIVEIRA et al., 2003).

Com esse propósito, guias alimentares foram desenvolvidos no Brasil para orientar pais e responsáveis em relação ao consumo e preferências alimentares do público infantil, priorizando a amamentação e a introdução e manutenção do consumo de uma alimentação saudável, sendo o mais recente elaborado por Philippi et al. (2003).

A obesidade infantil, por ser fator de risco para o desenvolvimento de outras doenças, tem se destacado nas prevenções voltadas à saúde pública (MELLO et al., 2004). De acordo com Aquino et al. (2002), o poder aquisitivo das famílias está fortemente condicionado à disponibilidade, quantidade e qualidade dos alimentos que farão parte da alimentação infantil.

O ambiente escolar, por também prover o acesso aos alimentos, também aparece como um espaço privilegiado para o desenvolvimento de ações de melhoria e das condições de saúde e do estado nutricional das crianças (SCHIMITZ et al., 2008). Porém, a grande oferta de alimentos ricos em gorduras - como bolachas, salgadinhos chips, sorvetes e refrigerantes - nas cantinas ou nas máquinas de vendas de alimentos presentes nas escolas, acaba por fazer com que muitos alunos deixem de consumir o lanche do programa escolar (LEITE et al., 2012).

Estudos de consumo individual do público infantil tornam-se necessários, visto que a disponibilidade desse grupo de alimentos nos dois ambientes está associada ao risco de obesidade (CANNELA et al., 2014). 
Visto que muitos pais desconhecem os diferentes processos pelos quais o alimento passa antes de chegar a seus filhos e de forma equivocada interpretam que a alimentação esteja sendo saudável, mesmo sendo esta formada em grande parte por produtos ultraprocessados, o presente estudo busca investigar o consumo de alimentos conforme seu grau de processamento por crianças de escolas privadas. Ademais, busca-se investigar se há fatores que distraem a criança no ato de se alimentar e avaliar o fracionamento das refeições.

\section{MÉTODOS}

Foi realizado um estudo exploratório de caráter transversal, cujos dados foram coletados entre junho e agosto de 2016 em escolas privadas da região Metropolitana de São Paulo.

A amostra foi constituída por crianças de 2 a 7 anos frequentadoras dessas instituições. Os pais e responsáveis responderam um questionário elaborado pelo Sistema de Vigilância Alimentar e Nutricional (SISVAN) denominado "Marcadores do Consumo Alimentar" (BRASIL, 2015), elaborado com base na nova proposta do Guia Alimentar para a População Brasileira (BRASIL, 2014). Uma vez que o SISVAN acredita que a alimentação e a nutrição se

\section{RESULTADOS E DISCUSSÃO}

De acordo com os critérios descritos na metodologia, participaram do estudo 70 crianças de ambos os gêneros, sendo 39 meninas $(55,71 \%)$.

Na tabela 1, pode-se observar o número de refeições que as crianças fizeram ao longo do dia anterior, totalizando 334 refeições. Foi constituem como fatores determinantes e condicionantes da saúde de um indivíduo, o questionário utilizado tem como propósito identificar padrões de alimentação e comportamento saudáveis e não saudável com base no grau de processamento dos alimentos, identificando a qualidade e comportamentos de risco do grupo estudado (BRASIL, 2004).

Foram explicados os objetivos do estudo, as questões éticas envolvidas e o preenchimento do questionário orientado pela pesquisadora, sendo este realizado pessoalmente ou com o auxílio de ferramentas tecnológicas. As variáveis foram analisadas por meio de frequências em número e porcentagem e apresentadas de forma descritiva, por meio de tabelas e gráficos.

Foram respeitadas as diretrizes e normas que regulamentam as pesquisas envolvendo seres humanos, sendo mantidos o anonimato e confidencialidade dos dados e garantidos o direito dos participantes em desistir da pesquisa a qualquer momento, tendo os responsáveis assinado o termo de consentimento livre e esclarecido (BRASIL, 2016). O estudo foi submetido à aprovação do Comitê de Ética em Pesquisa da Universidade Presbiteriana Mackenzie e aprovado sob número CAAE 50839915.9.0000.0084.

observado que houve um consumo adequado, se considerado os resultados do SISVAN (BRASIL, 2015), visto que as três principais refeições (café da manhã, almoço e jantar) foram realizadas por todas as crianças. 
Tabela 1 - Analise dos totais de refeições feitas ao longo do dia anterior. São Paulo, 2016.

\begin{tabular}{ccc}
\hline Refeições realizadas & N & \% \\
\hline Café da manhã & 70 & 100 \\
Lanche da manhã & 45 & 64 \\
Almoço & 70 & 100 \\
Lanche da tarde & 61 & 87 \\
Jantar & 70 & 100 \\
Ceia & 18 & 26
\end{tabular}

Fonte: elaborado pelos autores.

Apesar de ser positivo, tal resultado também remete a necessidade de uma necessidade calórica maior por esse público por serem muito ativos e estarem em fase de crescimento, além de o Guia Alimentar incentivar o consumo de pequenas refeições intercaladas às principais para que não haja um desequilíbrio energético e de nutrientes (BRASIL, 2014).

Ademais, visto que não são todas as crianças que realizam as refeições intermediárias, observa-se a necessidade do consumo de um desjejum adequado em todos os dias da semana, tanto para manutenção do peso, se composta por alimentos ricos em açúcar e gordura, quanto para se prevenir uma deficiência de micronutrientes, uma vez que conterá alimentos ricos em vitaminas e minerais, como o cálcio (PINTO et al., 2011).

Na infância, o descobrimento de sabores, texturas e cores, experiências sensoriais que irão influenciar o padrão alimentar a ser adotado pela criança na vida adulta. De tal forma, é essencial incentivar e se incorporar uma alimentação qualitativa e quantitativamente adequada, que garanta o fornecimento de energia e nutrientes necessários para o crescimento, desenvolvimento e manutenção da saúde da criança (MENEGAZZO, 2011).

Por ser um local no qual as crianças passam considerável tempo do dia, o ambiente escolar é fundamental para a determinação desses hábitos, pois além do fornecimento das refeições também há influências advindas do convívio com educadores, cuidadores e com outras crianças, devendo, portanto, oferecer refeições nutricionalmente adequadas, incentivando e inserindo também conceitos de alimentação saudável na prática educativa (ALBIERO et al., 2007).

O comportamento alimentar se caracteriza pelo o que se come, quanto, como, quando, onde e com quem se come, bem como a seleção de alimentos e os aspectos referentes ao preparo da comida. Os pré-escolares, especificamente, devem ser expostos a alimentos saudáveis para que, ao gostarem e terem o hábito de consumo, refutem os que não lhes foram apresentados por considerarem não palatáveis (ASSAO et al., 2008).

Além disso, o comportamento alimentar está associado a atributos socioculturais, 
envolvendo aspectos subjetivos individuais e coletivos relacionados ao comer e à comida (CASTRO, 2016).

Assim sendo, apesar de recomendado um ambiente tranquilo e sem distração e sempre que possível em companhia para o momento de refeição da criança, de acordo com a figura 1 , $46 \%$ realiza refeições com alguma distração tecnológica, como TV, games e celulares, o que impacta na percepção da quantidade e tamanho das porções consumidas, comprometendo a saciedade. (BRASIL, 2015).

Figura 1 - Comportamento alimentar de crianças de 2 a 7 anos em relação ao hábito de se alimentar na presença de equipamentos tecnológicos. São Paulo, 2016.

$46 \%$

$$
34 \%
$$

Fonte: Elaborado pelos autores

Visto que as porções dos alimentos podem contribuir para o desenvolvimento de DCNT, consideradas por Menegazzo (2011) como um desafio à segurança alimentar e nutricional, o recomendado para crianças de dois a seis anos é o equivalente a dois terços do tamanho da porção do alimento sugerido aos adultos por grupo alimentar, com exceção para o grupo do leite, cujo porcionamento deve ser o mesmo (BARBOSA, 2006).

Além das distrações observadas, deve ser considerada para o desenvolvimento da obesidade a disponibilidade de alimentos processados e ultraprocessados no ambiente doméstico, devido a sua maior densidade energética (MENEGAZZO, 2011). Como prevenção, observa-se a prática de atividades físicas regular nessa faixa etária, além de uma educação alimentar e nutricional para as crianças e orientação aos pais para uma aquisição e promoção mais adequada de alimentos (ALBIERO et al., 2007; BARBOSA, 2006).

Na tabela 2, pode-se observar o consumo de alimento in natura e minimamente processados e o percentual de ingestão destes alimentos ao longo do dia anterior. 
Tabela 2 - Consumo de alimentos in natura e minimamente processados por crianças de 2 a 7 anos, São Paulo, 2016.

\begin{tabular}{lccccc}
\hline & Alimentos & Sim & \% & Não & \% \\
\hline Feijão & 61 & 87,2 & 9 & 12,9 \\
Frutas frescas & 44 & 62,9 & 26 & 37,1 \\
Verduras e/ou legumes & 49 & 70 & 21 & 30
\end{tabular}

Fonte: Elaborado pelos autores.

O consumo de feijão foi satisfatório $(87,2 \%)$. Trata-se de uma leguminosa rica em proteína, fibra, vitaminas $\mathrm{B}$, cálcio e zinco. Se consumido com o arroz, tornará a refeição completa em proteínas de alto valor biológico, caracterizando esta opção tipicamente brasileira como benéfica à saúde, além de constituída de alimentos minimamente processados, cujo consumo é estimulado pelo Guia alimentar para a população brasileira (BRASIL, 2014). Ademais, seu consumo é recomendado ao menos uma vez ao dia, por ser uma grande fonte de ferro em um contexto em que a anemia ferropriva é prevalente em países tanto desenvolvidos quanto em desenvolvimento (SALDIVA et al., 2010).

O consumo de frutas frescas surpreendeu positivamente $(62,9 \%)$, visto que não foram considerados os sucos de frutas. Seu consumo deve ser de três porções diárias, pois são ricas em vitaminas, minerais e fibras alimentar, principalmente nos lanches intermediários e nas sobremesas, variando-se o tipo e respeitando-se a sazonalidade (BRASIL, 2015).

De acordo com a Sociedade Brasileira de Pediatria (2012), o consumo de frutas, verduras e legumes deve ser incentivado ao público infantil devido à formação das preferências alimentares e por serem o alicerce para uma alimentação nutricionalmente balanceada, saborosa, culturalmente apropriada e geradora de um sistema alimentar socialmente e ambientalmente sustentável (BRASIL, 2014).

Como a literatura relata ser de difícil aceitação e inserção na dieta infantil, o consumo de verduras $(70 \%)$ também foi considerado relevante, principalmente por não terem sido considerados tubérculos; devem estar presentes nas refeições devido a sua qualidade nutricional e baixa densidade calórica e prevenção de DCNT (BRASIL, 2014; JAIME et al., 2007).

Na tabela 3, pode-se observar um alto consumo de alimento processados e ultraprocessados e o percentual de ingestão destes alimentos ao longo do dia anterior. 
Tabela 3 - Consumo de alimentos processados e ultraprocessados por crianças de 2 a 7 anos, São Paulo, 2016.

\begin{tabular}{ccccc}
\hline Alimentos consumidos & Sim & \% & Não & \% \\
\hline Hamburguer e/ou embutidos & 36 & 51,4 & 34 & 48,6 \\
Bebidas adoçadas & 57 & 81,4 & 13 & 18,6 \\
salgados & & & 48,6 \\
Biscoito recheado, doces ou guloseimas balas, pirulitos, \\
chiclete, caramelo, gelatina
\end{tabular}

Fonte: Elaborada pelos autores.

Mais da metade das crianças $(51,4 \%)$ consumiu hambúrguer e embutidos no dia anterior à coleta, sendo estes representados por presunto, mortadela, salame, lingüiça e salsicha. Os pais e responsáveis pela alimentação da criança devem ser orientados a evitar esses alimentos devido à alta quantidade de gordura saturada e sódio e substituí-los por carnes brancas (peixe e frango), vermelhas e ovos, fontes de proteínas, vitaminas e minerais (BRASIL, 2015).

O consumo de refrigerantes, sucos e águas de coco industrializados, xaropes de guaraná/groselha ou sucos de fruta com adição de açúcar, foi observado um alto consumo (81,42\%), assim como de doces, guloseimas e/ou biscoito recheados observados $(77,14 \%)$, pode ser prejudicial à saúde da criança devido a concentração exorbitante de açúcares e, no caso dos biscoitos, de gorduras. Também pode desestimular a ingestão de frutas in natura, sucos de frutas naturais e até mesmo o consumo de água (BRASIL, 2015).

Em paralelo ao consumo de açúcar, o de gorduras e sódio advindos de macarrões instantâneos, salgadinhos de pacotes e biscoitos salgados relatado por $51,4 \%$ dos pais no dia anterior é preocupante devido a esses produtos poderem desencadear DCNT. Ademais, em relação ao consumo de sódio, excedem os níveis de ingestão recomendados pela Organização Mundial da Saúde (2013), como a ingestão recomendada não deve exceder $2 \mathrm{~g}$ ao dia, devese limitar o consumo de alimentos processados devido a sua quantidade ser superior ao intrinsecamente presente nos alimentos in natura.

Devido à facilidade de consumo e ao cenário atual, com a industrialização e maior participação da mulher no mercado de trabalho, alimentos ultraprocessados são inferiores nutricionalmente e contribuem não somente com ingredientes maléficos à saúde humana e com alta densidade calórica, mas na diminuição da ingestão de alimentos ricos em fibra e em potássio (LOUZADA et al., 2015; MONTEIRO, 2011).

Em relação ao público infantil, observouse forte consumo de alimentos industrializados, o que tende a impactar negativamente na qualidade nutricional da dieta e da saúde (MONTEIRO et al., 2016).

\section{CONCLUSÃO}

A alimentação dos avaliados em relação ao consumo de produtos processados e 
ultraprocessados se mostrou um fator preocupante tendo em vista suas repercussões negativas a saúde futura.

A maioria consome alimentos in natura $\mathrm{e}$ minimamente processados isto é um dado positivo, mas tenho que levar em consideração que este dado se refere apenas ao dia anterior, não há como mensurar se este dado se refere à alimentação diária.

Conclui-se que são necessárias ações para conhecimento e conscientização sobre estes tipos de alimentos e como podem mudar este hábito no dia a dia das crianças para melhora na qualidade de vida.

\section{REFERÊNCIAS}

ALBIERO, K.A. et al. Formação e desenvolvimento de hábitos alimentares em crianças pela educação nutricional. Rev. Nutrição em Pauta, v.15, n. 82, p. 17-21, jan./fev, 2007.

AQUINO, R.C. et al. Consumo infantil de alimentos industrializados e renda familiar na cidade de São Paulo. Rev. Saúd. Pública. v.36, n.6, p.655-660, 2002.

ASSAO, T.Y. et al. Alimentação saudável: percepções dos educadores de instituições infantis. Rev. Bras. Crescimento Desenvolv Hum, v.18, n. 2, p. 1261134, 2008

BARALDI, L.G, et al. Alimentos ultraprocessados e perfil nutricional da dieta no Brasil. Rev. Saúde Pública. v. 49, p. 45, 2015.

BARBOSA, R.M.S.; et al. Guias alimentares para crianças: aspectos históricos e evolução. Rev. Nutr. Campinas. v. 19, n.2, p. 255-263, 2006.

BIELEMANN, M. R. et al. Consumo de alimentos ultraprocessados e impacto na dieta de adulto jovens. Rev. Saúde Pública. v. 49, p.28, 2015.
BRASIL. Ministério da Saúde. Secretaria de atenção à saúde. Política nacional de promoção da saúde. Brasília: Ministério da Saúde, 2007.

BRASIL. Ministério da Saúde. SISVAN - Notas

Técnicas. Secretaria de Atenção à Saúde. MS/SAS/Departamento de Atenção Básica. Coordenação Geral da Politica de Alimentação e Nutrição. Brasília: MS; 2004. Disponível em: <http://tabnet.datasus.gov.br/cgi/SISVAN/CNV/notas _sisvan.html >. Acesso em: 14 out. 2016.

BRASIL. Ministério da Saúde. Secretaria de Atenção à Saúde. Guia Alimentar para a população brasileira: promovendo a alimentação saudável. Brasília: MS; 2014. Disponível em: <http://189.28.128.100/dab/docs/portaldab/publicacoe s/guia_alimentar_populacao_brasileira.pdf $>$. Acesso em: 04 abr. 2016.

BRASIL. Ministério da Saúde. Secretária de Atenção a Saúde. Orientações para Avaliação de Marcadores de Consumo Alimentar na Atenção Básica. Brasília: MS; 2015. Disponível em: <http://bvsms.saude.gov.br/bvs/publicacoes/marcador es_consumo_alimentar_atencao_basica.pdf $>$ Acesso em: 26 mai. 2016.

BRASIL. Diário Oficial da união (BR). Resolução n 510, de 07 de abril de 2016. Disponível em: <http://conselho.saude.gov.br/resolucoes/2016/Reso51 $\underline{0 . p d f}>$. Acesso em: 10 jun. 2016.

CASTRO, L.R. A Educação Alimentar e Nutricional no processo de mudança de comportamento: metodologia e amor. Rev. Eletronica Puc-RS. v. 9, n. $1,2016$.

GALLIAN, D.M.C. A desumanização do comer Estudos Avançados, São Paulo, v. 21, n. 60, p. 179184, 2007.

JAIME, C.P. et al. Educação nutricional e consumo de frutas e hortaliças: ensaio comunitário controlado. Rev. Saúde Pública. v.39, n. 4, p.530-540, 2007.

LEITE, F.H.M. et al. Oferta de alimentos processados no entorno de escolas públicas em área urbana. Rev. Jornal de pediatria, v.88, n.4, p.328-34, 2012.

LOUZADA, M.L.C. et al. Impacto de alimentos ultraprocessados sobre o teor de micronutrientes da dieta do Brasil. Rev. Saúde Pública. v.49, n.45, p.1$8,2015$. 
MARTINS, B.A.P. et al. Participação crescente de produtos ultraprocessados na dieta brasileira (1987 2009). Rev. Saúde Pública. v. 49, n.4, p. 656-65, 2013.

MELLO, D.E.; et al. Obesidade infantil: Como podemos ser eficazes? Jornal de pediatria. v. 80, n.3, p.173-182, 2004.

MENEGAZZO, M. et al. Avaliação qualitativa das do cardápio de centros de educação infantil. Rev.

Nutrição Campinas. v. 24, n. 2, p. 24-251, 2011.

MONTEIRO, C.A. et al. Increasing consumption of ultra-processed foods and likely impact on human health: evidence from Brazil. Public Health Nutr. n. 14, v. 1, p. 5-13, 2011.

MONTEIRO, C.A. et al., Alimentos ultraprocessados e perfil nutricional da dieta no Brasil. Rev Saúde Pública. p 49:38, 2015.

MONTEIRO, C.A. et al. Classificação NOVA dos alimentos. Rev. Higiene Alimentar. Pub. em World Nutrition, v. 7, n. 13, 2016.

OLIVEIRA, M. C. F. et al. Alimentação saudável do pré- escolar. In: SILVA, M. M. S.; CAMPOS, M. T. F. S. (Ed.). Segurança alimentar e nutricional na atenção básica em saúde. Viçosa, MG: UFV, p. 117132, 2003.

PINTO, C.K.O., et al. A influência do desjejum no peso corporal de crianças e adolescentes. Rev. Nutr em Pauta, v. 2, n.35, p. 36-39, 2011.

PHILIPPI, S.T. et al. Pirâmide alimentar para crianças de 2 e 3 anos. Rev. Nutr. Campinas. v. 16, n.1. p. 5-19, 2003.

SALDIVA, S. R. D. M; et al. Avaliação antropométrica e consumo alimentar em crianças menores de cinco anos residentes em um município da região do semiárido nordestino com cobertura parcial do programa bolsa família. Rev. de Nutrição, v. 23, n. 2, p. 221-229, 2010.

SOCIEDADE BRASILEIRA DE PEDIATRIA. Manual de orientação do departamento de nutrologia: alimentação do lactente ao adolescente, alimentação na escola, alimentação saudável e vinculo da mãe-filho, alimentação saudável e prevenção de doenças, segurança alimentar. Rio de Janeiro, 2012.
WHO. Organização Mundial da Saúde. Child Health and Development. Evidence for the ten steps to successful breastfeeding. Geneva, 2000. Disponível em:

<http://whqlibdoc.who.int/publications/2004/9241591 544 eng.pdf $>$. Acesso em: 05 out. 2016.

WHO. Organização Mundial da Saúde. OMS emite novas orientações sobre dietética de sal e potássio. Geneva, 2013. Disponível em:

<http://www.who.int/mediacentre/news/notes/2013/sal t_potassium 20130131/en/>. Acesso em: 05 out. 2016.

\section{Iracy Marles Godim Fabiano}

Graduada do curso de Nutrição do Centro de Ciências Biológicas e da Saúde da Universidade Presbiteriana Mackenzie

\section{Daniela Maria Alves Chaud}

Nutricionista, mestre e doutora em Ciências Aplicadas à Pediatria, professora do curso de Nutrição do Centro de Ciências Biológicas e da Saúde da Universidade Presbiteriana Mackenzie

Edeli Simioni de Abreu
Nutricionista, mestre e doutora em Saúde Pública
pela Universidade de São Paulo (USP).

